\title{
Rapid Polymyxin/Pseudomonas NP test for rapid detection of polymyxin susceptibility/resistance in Pseudomonas aeruginosa
}

\author{
Mustafa Sadek ${ }^{1} \cdot$ Camille Tinguely $^{1} \cdot$ Laurent Poirel $^{1,2,3} \cdot$ Patrice Nordmann ${ }^{1,2,3,4}$
}

\begin{abstract}
Polymyxins are becoming an added value for treating infections due to multidrug-resistant Pseudomonas aeruginosa. Therefore, the Rapid Polymyxin/Pseudomonas NP test was developed for the identification of polymyxin resistance in $P$. aeruginosa isolates. This test detects the bacterial growth in a medium containing a defined concentration of colistin. Formation of basic metabolites is visually detected by yellow to purple/violet color change of the bromocresol purple $\mathrm{pH}$ indicator. The test performance was evaluated by using 50 colistin-resistant and colistin-susceptible $P$. aeruginosa isolates, among which 10 were colistin resistant. The sensitivity and specificity of the test were found to be $100 \%$ (CI95 65.5$100 \%$ ) and 95\% (CI95 81.8-99.1\%), respectively, by comparison with broth microdilution as the reference method. The Rapid Polymyxin/Pseudomonas NP test is easy to perform, specific and sensitive, allowing rapid visual observation of results without the requirement any special reading equipment, and results are obtained in $3 \mathrm{~h}$.
\end{abstract}

Keywords Colistin $\cdot$ Rapid diagnostic test $\cdot$ Pseudomonas aeruginosa $\cdot$ Susceptibility testing

\section{Introduction}

Pseudomonas aeruginosa belongs to the ESKAPE group of pathogens (Enterococcus faecium, Staphylococcus aureus, Klebsiella pneumoniae, Acinetobacter baumannii, $P$. aeruginosa, and Enterobacter species). It is one of the most important bacterial species in clinical settings in particular as a source of bacterial pneumonia in intensive care patients $[1,2]$. Carbapenem-resistant $P$. aeruginosa isolates are increasingly reported in health care facilities that may lead to fatal outcomes due to limited therapeutic options [3-5]. The Center for Diseases Control of Atlanta in the USA and the World

Patrice Nordmann

patrice.nordmann@unifr.ch

1 Medical and Molecular Microbiology, Faculty of Science and Medicine, University of Fribourg, Chemin du Musée 18, 1700 Fribourg, Switzerland

2 INSERM European Unit (IAME), University of Fribourg, Fribourg, Switzerland

3 Swiss National Reference Center for Emerging Antibiotic Resistance (NARA), University of Fribourg, Fribourg, Switzerland

4 Institute for Microbiology, University of Lausanne and University Hospital Centre, Lausanne, Switzerland
Health Organization classified the carbapenem-resistant $P$. aeruginosa among the most serious pathogens exhibiting multidrug resistance $[6,7]$.

Polymyxins are considered as a last resort treatment for treating multidrug-resistant $P$. aeruginosa infections [8]. Owing to the occurrence of colistin resistance in this species, reliable and rapid techniques for testing colistin susceptibility are needed to optimize antibiotic stewardship. The standard reference technique for determining polymyxin susceptibility in Gram-negative bacteria is the determination of the corresponding MIC by the broth microdilution method (BMD) [9], which is however time-consuming $(24 \mathrm{~h})$ and is impractical in routine microbiology. Other techniques for determining susceptibility to polymyxins such as disk diffusion and E-test are not recommended due to high rates of false-susceptibility results. Recently, we have developed the Rapid Polymyxin NP test that identifies colistin-susceptible/colistin-resistant isolates in $2 \mathrm{~h}$ for Enterobacterales [10]. This test is based on the visualization of glucose metabolization and consequently production of acidic components in the presence of a $\mathrm{pH}$ indicator. Here, we have developed a rapid test (the Rapid Polymyxin/Pseudomonas NP test) for identification of polymyxin resistance in $P$. aeruginosa. This test detects the bacterial growth in a medium supplemented with a defined concentration of colistin. Taking in account the production of alkaline 
products during $P$. aeruginosa metabolism, formation of basic metabolites was visually detected by yellow to purple/violet color change of the bromocresol purple taken as the $\mathrm{pH}$ indicator. The test is cost-effective, easy to perform, and interpreted through a rapid visual observation in $3 \mathrm{~h}$. The objective of this study was to develop this novel test by comparison with the BMD method using a collection of colistinsusceptible and colistin-resistant $P$. aeruginosa clinical isolates.

\section{Materials and methods}

Bacterial strains This study was carried out using 50 wellcharacterized $P$. aeruginosa clinical isolates. Ten out of the $50 P$. aeruginosa isolates were colistin resistant according to the results of the BMD testing (Table 1). The colistinsusceptible $P$. aeruginosa ATCC 27853 strain and the colistin-resistant $P$. aeruginosa R409 strain were used as negative and positive controls for the determination of MIC of colistin, respectively.

Antimicrobial susceptibility testing The BMD was performed, using homemade panels, for determination of MICs of colistin in cation-adjusted Mueller-Hinton broth (Bio-Rad, MarnesLa-Coquette, France, ref. 69444), and results were interpreted according to the EUCAST/CLSI joint guidelines [9]. Isolates were considered susceptible when MICs of colistin were $\leq 2 \mathrm{mg} / \mathrm{L}$ and resistant when MICs were $>2 \mathrm{mg} / \mathrm{L}$ [9]. Colistin sulfate (Sigma-Aldrich, St. Louis, MO, USA) were tested over a range of dilutions $(0.125-128 \mathrm{mg} / \mathrm{L})$. These antibiotic powders can be stored at $4{ }^{\circ} \mathrm{C}$ before their use, whereas diluted polymyxin solutions may be kept at $-20^{\circ} \mathrm{C}$ for up to 1 year.

\section{Rapid Polymyxin/Pseudomonas NP test}

We first compared different parameters using a colistinsusceptible isolate ( $P$. aeruginosa ATCC 27853) and a colistin-resistant isolate ( $P$. aeruginosa $\mathrm{R}-409)$. These parameters included bacterial inoculum $(0.5,1.5$, and $3.5 \mathrm{McF}$ arland standards), colistin concentration (1.75, 2, 2.5, 3, 3.5, $4 \mathrm{mg} / \mathrm{L})$, and time of incubation (1, 2, and $3 \mathrm{~h}$ ). After comparison of the results with different parameters, all experiments were performed in triplicate by two different persons with the optimal conditions obtained, as described below.

Preparation of solutions For stock solutions of polymyxins, colistin sulfate tablets (Mast Diagnostics, Merseyside, UK) were dissolved into Mueller-Hinton (MH) solution (BioRad, Marnes-La-Coquette, France, ref. 69444) in glass tubes to obtain a concentration of $0.256 \mathrm{mg} / \mathrm{mL}$. These tablets can be stored at $4{ }^{\circ} \mathrm{C}$ before use, and the diluted polymyxin solutions can be kept at $-20{ }^{\circ} \mathrm{C}$ for 1 year.

The Rapid Polymyxin/Pseudomonas NP solution To prepare $250 \mathrm{~mL}$ of the Rapid Polymyxin/Pseudomonas NP solution, the culture medium and the $\mathrm{pH}$ indicator were mixed in a glass bottle as follows: $6.25 \mathrm{~g}$ of MHB-CA powder (cation-adjusted Mueller-Hinton broth, Bio-Rad, Marnes-La-Coquette, France, ref. 69444), $0.00625 \mathrm{~g}$ of bromocresol purple (SigmaAldrich), and $225 \mathrm{~mL}$ of distilled water. Several $\mathrm{pH}$ indicators were tested before retaining bromocresol purple that gave the optimal results (data not shown). The $\mathrm{pH}$ of the solution was adjusted to $5.25-5.4$ by adding drops of $1 \mathrm{M} \mathrm{HCl}$. Then, the final volume was adjusted to $250 \mathrm{~mL}$, and the solution was autoclaved at $121{ }^{\circ} \mathrm{C}$ for $15 \mathrm{~min}$. The final concentrations in the Rapid Polymyxin/Pseudomonas NP solution were consequently $2.5 \%$ of MHB-CA powder and $0.0026 \%$ of bromocresol purple indicator. This Rapid Polymyxin/ Pseudomonas NP solution can be kept at $4{ }^{\circ} \mathrm{C}$ for 1 week or at $-20{ }^{\circ} \mathrm{C}$ for 1 year. This solution must be pre-warmed at $37{ }^{\circ} \mathrm{C}$ before use to prevent growth delay and therefore a delayed color change. Just before performing the experiment, colistin is added to the Rapid Polymyxin/Pseudomonas NP solution and mixed into sterile glass tubes to obtain the Rapid Polymyxin/Pseudomonas NP solution containing a colistin concentration of $4 \mathrm{mg} / \mathrm{L}$. As an example, $15.6 \mu \mathrm{L}$ of colistin stock solution at $0.256 \mathrm{mg} / \mathrm{mL}$ was added to $1 \mathrm{~mL}$ of Rapid Polymyxin/Pseudomonas NP solution for the testing of one clinical isolate and along with negative and positive controls.

Bacterial inoculum preparation For each isolate to be tested including the positive and negative controls, we prepared a standardized bacterial inoculum by using freshly obtained (overnight) bacterial colonies grown on UriSelect 4 agar plates (or Muller-Hinton agar plates). The bacterial colonies were resuspended into $5 \mathrm{~mL}$ of sterile $\mathrm{NaCl}$ $(0.85 \%)$ to obtain a 3.0-3.5 McFarland standard optical density $\left(\sim 10^{9} \mathrm{CFU} / \mathrm{mL}\right)$. This corresponds to about a $10-\mu \mathrm{L}$ full loop of bacterial colonies diluted in $10 \mathrm{~mL}$ of $\mathrm{NaCl} 0.85 \%$. We used as positive control (resistant isolate) P. aeruginosa R409 and as negative control (susceptible isolate) P. aeruginosa ATCC27853 (R1496). The bacterial suspensions should be used within $15 \mathrm{~min}$ of preparation and for no longer than $1 \mathrm{~h}$ after preparation, as recommended by the EUCAST guidelines for susceptibility testing [9].

Tray inoculation Inoculation and reading were performed similarly to what is recommended for the optimized Rapid Polymyxin NP test. We performed the testing in a 96-well polystyrene microplate (round base, with lid, sterile, Sarstedt Str. 1, D-51588 Nuembrecht, Germany, ref. 
Table 1 Rapid Polymyxin/ Pseudomonas NP test for polymyxin-resistant and polymyxin-susceptible P. aeruginosa

\begin{tabular}{|c|c|c|c|c|c|c|}
\hline \multirow[t]{2}{*}{ Strain } & \multirow[t]{2}{*}{ Origin } & & \multirow[t]{2}{*}{ Phenotype ${ }^{a}$} & \multirow{2}{*}{$\begin{array}{l}\text { BMD MIC } \\
\text { colistin }(\mathrm{mg} / \mathrm{L})\end{array}$} & \multicolumn{2}{|c|}{ Rapid Polymyxin/Pseudomonas NP test } \\
\hline & & & & & Result & $\begin{array}{l}\text { Discrepancy with BMD } \\
\text { MIC colistin result }\end{array}$ \\
\hline R1496 & USA & Reference & $\mathrm{S}$ & 0.125 & Negative & - \\
\hline R104 & France & Clinical & $\mathrm{S}$ & 0.125 & Negative & - \\
\hline R49 & France & Clinical & $\mathrm{S}$ & 0.125 & Negative & - \\
\hline R70 & France & Clinical & $\mathrm{S}$ & 0.125 & Negative & - \\
\hline R96 & France & Clinical & $\mathrm{S}$ & 0.125 & Negative & - \\
\hline R47 & France & Clinical & $\mathrm{S}$ & 0.25 & Negative & - \\
\hline R66 & France & Clinical & $\mathrm{S}$ & $<0.125$ & Negative & - \\
\hline R64 & France & Clinical & S & 0.25 & Negative & - \\
\hline R76 & France & Clinical & $\mathrm{S}$ & 0.5 & Negative & - \\
\hline R3407 & France & Clinical & $\mathrm{S}$ & 0.125 & Negative & - \\
\hline R3409 & France & Clinical & $\mathrm{S}$ & 0.125 & Negative & - \\
\hline R3408 & France & Clinical & $\mathrm{S}$ & 0.125 & Negative & - \\
\hline R3410 & France & Clinical & $\mathrm{S}$ & 0.125 & Negative & - \\
\hline R124 & France & Clinical & $\mathrm{S}$ & 0.125 & Negative & - \\
\hline R126 & France & Clinical & $\mathrm{S}$ & 0.125 & Negative & - \\
\hline R127 & France & Clinical & $\mathrm{S}$ & 0.125 & Negative & - \\
\hline R1202 & Greece & Clinical & $\mathrm{S}$ & 0.125 & Negative & - \\
\hline R196 & France & Clinical & $\mathrm{S}$ & 0.125 & Negative & - \\
\hline R606 & France & Clinical & $\mathrm{S}$ & 0.125 & Negative & - \\
\hline R15 & France & Clinical & $\mathrm{S}$ & $<0.125$ & Negative & - \\
\hline R128 & France & Clinical & $\mathrm{S}$ & 0.5 & Positive & YES (ME) \\
\hline R3411 & France & Clinical & $\mathrm{S}$ & $<0.125$ & Negative & - \\
\hline R3412 & France & Clinical & $\mathrm{S}$ & $<0.125$ & Negative & - \\
\hline R3413 & France & Clinical & $\mathrm{S}$ & 0.125 & Negative & - \\
\hline R3425 & France & Clinical & $\mathrm{S}$ & 0.125 & Negative & - \\
\hline N142 & Switzerland & Clinical & $\mathrm{S}$ & 0.5 & Negative & - \\
\hline N143 & Switzerland & Clinical & $\mathrm{S}$ & 0.125 & Negative & - \\
\hline R608 & France & Clinical & $\mathrm{S}$ & 0.25 & Negative & - \\
\hline R419 & France & Clinical & $\mathrm{S}$ & 0.5 & Negative & - \\
\hline N144 & Switzerland & Clinical & $\mathrm{S}$ & 0.125 & Negative & - \\
\hline N158 & Switzerland & Clinical & $\mathrm{S}$ & 0.125 & Negative & - \\
\hline N167 & Switzerland & Clinical & $\mathrm{S}$ & 0.125 & Negative & - \\
\hline N192 & Switzerland & Clinical & $\mathrm{S}$ & 0.125 & Negative & - \\
\hline N214 & Switzerland & Clinical & $\mathrm{S}$ & 0.125 & Negative & - \\
\hline R105 & France & Clinical & $\mathrm{S}$ & 0.125 & Positive & YES (ME) \\
\hline N254 & Switzerland & Clinical & $\mathrm{S}$ & 0.125 & Negative & - \\
\hline N301 & Switzerland & Clinical & $\mathrm{S}$ & 0.125 & Negative & - \\
\hline N69 & Switzerland & Clinical & $\mathrm{S}$ & 0.125 & Negative & - \\
\hline N170 & Switzerland & Clinical & $\mathrm{S}$ & 0.125 & Negative & - \\
\hline R275 & France & Clinical & $\mathrm{S}$ & 0.125 & Negative & - \\
\hline R3813 & France & Clinical & $\mathrm{R}$ & $>128$ & Positive & - \\
\hline R3422 & France & Clinical & $\mathrm{R}$ & 8 & Positive & - \\
\hline R1197 & Turkey & Clinical & $\mathrm{R}$ & 8 & Positive & - \\
\hline R3419 & France & Clinical & $\mathrm{R}$ & 32 & Positive & - \\
\hline R3418 & France & Clinical & $\mathrm{R}$ & 16 & Positive & - \\
\hline R3421 & France & Clinical & $\mathrm{R}$ & 16 & Positive & - \\
\hline R3427 & France & Clinical & $\mathrm{R}$ & 16 & Positive & - \\
\hline R409 & France & Clinical & $\mathrm{R}$ & 8 & Positive & - \\
\hline R3420 & France & Clinical & $\mathrm{R}$ & 64 & Positive & - \\
\hline N76 & Switzerland & Clinical & $\mathrm{R}$ & 4 & Positive & - \\
\hline
\end{tabular}

$S$ susceptible, $R$ resistant, - no discrepancy, $M E$ major error, $B M D$ broth microdilution
82.1582.001). For each isolate, bacterial suspension was inoculated in parallel into 2 wells, with and without colistin, in separate wells. The following steps of the Rapid Polymyxin/Pseudomonas NP test were performed, as illustrated in Fig. 1:
Step 1: $150 \mu \mathrm{L}$ of colistin-free Rapid Polymyxin/ Pseudomonas NP solution was transferred to wells A1-A8.

Step 2: $\quad 150 \mu \mathrm{L}$ of the Rapid Polymyxin/Pseudomonas NP solution containing colistin $(4 \mathrm{mg} / \mathrm{L}$ to obtain a final 


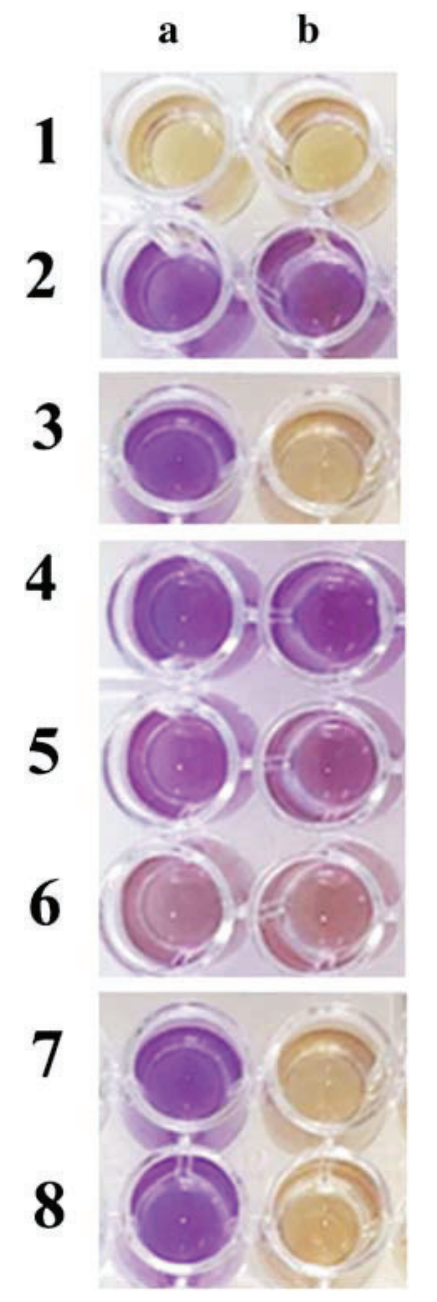

Fig. 1 Representative results of the Rapid Polymyxin/Pseudomonas NP test. Non-inoculated wells are shown as controls for possible and spontaneous color change (A1 and B1). Bacterial growth is evidenced by color change of the medium from yellow to purple or violet. The Rapid Polymyxin/Pseudomonas NP test was performed with a reference colistin-resistant $P$. aeruginosa isolate (A2 and B2) and with a reference colistin-susceptible $P$. aeruginosa isolate (A3 and B3) in a reaction without (first column, A) and with (second column, B) colistin at the defined concentration. The tested isolates (lines 4 to 6 ) that grew as well as in the absence and presence of colistin (wells A4-A6 and B4-B6), respectively) were colistin-resistant. The tested isolates (lines 7 to 8 ) that grew in the absence of colistin but not in its presence (wells A7-A8 and B7-B8), respectively) were colistin-susceptible isolates

concentration of $3 \mathrm{mg} / \mathrm{L}$ ) was transferred to wells B1-B8.

Step 3: $50 \mu \mathrm{L}$ of $\mathrm{NaCl} 0.85 \%$ was added to wells A1 and B1.

Step 4: $50 \mu \mathrm{L}$ of the colistin-resistant isolate suspension used as positive control was added to wells A2 and $\mathrm{B} 2$.

Step 5: $50 \mu \mathrm{L}$ of the colistin-susceptible isolate suspension used as negative control was added to wells A3 and B3.
Step 6: $50 \mu \mathrm{L}$ of the tested isolate suspension was added to wells A4-A8 and B4-B8.

The bacterial suspension was mixed with the rapid solution by pipetting up and down. The final concentration of bacteria was $\approx 10^{8} \mathrm{CFU} / \mathrm{mL}$ in each well, and the final concentration of colistin was $3.3 \mathrm{mg} / \mathrm{L}$.

Tray incubation The inoculated tray was incubated for up to $3 \mathrm{~h}$ at $35 \pm 2{ }^{\circ} \mathrm{C}$ in ambient air.

Tray reading Based on our previous experience of development of several rapid diagnostic tests $[10,11]$, We visually inspected the tray (checked for no spontaneous color change) after $10 \mathrm{~min}$ and then every hour for $3 \mathrm{~h}$. The test was considered positive (i.e., purple or violet of the culture medium, indicating polymyxin resistance if the tested isolate grew in the presence of colistin), and negative (i.e., yellow or yellowish green of the culture medium, indicating no growth and consequently polymyxin susceptibility) if the tested isolate did not grow in the presence of colistin. We considered the test result interpretable if the following 4 conditions were met: (1) both wells (A1 and B1) with $0.85 \% \mathrm{NaCl}$ and without bacterial suspension remained unchanged (absence of medium contamination); (2) the colistin-free wells A2-A8 with bacterial suspensions turned from yellow to purple or violet, confirming the growth of the isolate; (3) the well B2 with the colistin-resistant reference bacterial suspension (positive control) gave a positive result (purple/violet), confirming the viability of the isolate in the presence of colistin; and (4) the well B3 with the colistin-susceptible reference bacterial suspension (negative control) gave negative results (remaining yellow) confirming the absence of growth of this isolate. The tested isolates that grew in the absence and the presence of colistin (wells A4-A6 and B4-B6, respectively) were therefore reported to be colistin-resistant. The tested isolates that grew in the absence but did not grow in the presence of colistin (wells A7-A8 and B7-B8, respectively) were therefore reported as colistin-susceptible (Fig. 1).

The test result was positive when the well containing colistin (B2) and the isolate to be tested turned from yellow to purple or violet, both giving the exact same color as the well without colistin (A2), confirming the growth in the presence of colistin (colistin resistance) (Fig. 1). The test result was negative when the well containing colistin (B3) with the isolate to be tested remained yellow (unchanged color) (Fig. 1). Results were also interpreted by a technician in a blinded way.

Data analysis The results obtained with the Rapid Polymyxin/ Pseudomonas NP test was compared to those obtained with the reference BMD method. Briefly, discrepancies were determined for each method to assess the performances of the test 
to detect colistin resistance. Errors (very major errors [VME] and major errors $[\mathrm{ME}]$ ) were calculated as described previously [12]. A VME was considered when isolates were categorized as susceptible using the Rapid Polymyxin/Pseudomonas NP test, being however resistant by the BMD method (falsesusceptible result). A ME was considered when isolates were found to be resistant using the Rapid Polymyxin/ Pseudomonas NP test, being however susceptible by using the BMD method (false-resistant result).

\section{Results}

Among the fifty $P$. aeruginosa isolates included to assess the performance of the Rapid Polymyxin/Pseudomonas NP test (Table 1), 40 were colistin susceptible (MICs of colistin ranging from less than $<0.125$ to $0.5 \mathrm{mg} / \mathrm{L}$ ) and ten isolates were resistant (MICs of colistin ranging from 4 to $128 \mathrm{mg}$ / $\mathrm{L})$, according to the results of the BMD method. The 40 colistin-susceptible $P$. aeruginosa isolates gave negative results according to the Rapid Polymyxin/Pseudomonas NP test, except for two isolates (MICs of $0.125-0.5 \mathrm{mg} / \mathrm{L}$ ) which gave positive (false-positive) results (Table 1). The colistin-resistant $P$. aeruginosa isolates $(n=10)$ (MICs of colistin ranging from 4 to $128 \mathrm{mg} / \mathrm{L}$ ) were all correctly identified as colistin resistant by using the Rapid Polymyxin/Pseudomonas NP test (Table 1). Overall, two ME (false resistance) but no VME (false susceptibility) were observed. Therefore, a strong concordance was found between the results of the susceptibility test and of the Rapid Polymyxin/Pseudomonas NP test results, both for susceptible and resistant isolates (Table 1). Sensitivity (100\%, CI95 $65.5-100 \%)$ and specificity (95\%, CI95 81.8-99.1\%) of the test were therefore high compared with the BMD method. By reading the color change of the wells every hour, we determined that final results shall be best read at $3 \mathrm{~h}$ after incubation at $35 \pm 2{ }^{\circ} \mathrm{C}$ under an ambient atmosphere.

\section{Discussion}

This study showed that the Rapid Polymyxin/Pseudomonas NP test is a reliable technique for detecting polymyxin resistance in $P$. aeruginosa. Development of this test is based on detection of the bacterial growth in a medium containing a defined concentration of colistin. Detection of alkaline constituents in the growth medium (and not acidic components as for the Rapid Polymyxin NP for Enterobacterales) is made by adding a $\mathrm{pH}$ indicator, bromocresol purple, which color change occurs when the culture medium becomes more alkaline upon bacterial growth. This test is rapid, inexpensive, and easy to implement. It offers the possibility of detecting polymyxin resistance from bacterial cultures in $3 \mathrm{~h}$, which is at least $16 \mathrm{~h}$ earlier than with the reference BMD method, and faster than a test we previously developed (so-called Rapid ResaPolymyxin Acinetobacter/Pseudomonas NP test [11], requiring at least $4 \mathrm{~h}$ ). In addition, the Rapid Polymyxin/ Pseudomonas NP test requires a single methodological step while the Rapid ResaPolymyxin Acinetobacter/Pseudomonas NP test requires an additional one including growth period of time of $3 \mathrm{~h}$ and then addition of resazurin in the medium in another $1 \mathrm{~h}$ revelation period of time [11]. Even though a few discrepancies were observed (two ME), the sensitivity and specificity of the Rapid Polymyxin/Pseudomonas NP test were high (100\%, CI95 65.5-100\% and 95\%, CI95 81.8 $99.1 \%$, respectively), making it a potential useful technique for clinical laboratories. This test complements the Rapid Polymyxin NP test, which works with Enterobacterales but is not appropriate for non-fermenters such as $P$. aeruginosa. Altogether, such newly developed approach fits with what is expected from a rapid diagnostic test (besides accuracy with excellent sensitivity and specificity), namely optimal fastness and easiness.

Owing to the relatively small sample size of our collection which may be considered as a limitation, which is indicated by the wide the confidence interval (CI95) especially for sensitivity, further studies with a broader set of resistant isolates will be needed to further validate the accuracy of that test. In addition, validation of this test may be performed also with other Pseudomonas species, besides $P$. aeruginosa. The Rapid Polymyxin/Pseudomonas NP test may be useful for a firststep screening of polymyxin resistance, considering the diversity (and sometimes still unknown) mechanisms of resistance to polymyxins in P. aeruginosa. The turn-around time of such test is short, which may lead to improve the rapid implementation of adequate hygiene measures to control the spread of such multidrug-resistant bacteria. Ultimately, implementation of such test may significantly contribute to optimize the rationale use of polymyxins by differentiating rapidly infections due to polymyxin-susceptible or polymyxin-resistant $P$. aeruginosa isolates.

Acknowledgments We would like to thank Pr. P. Plésiat for providing us with a series of colistin-resistant $P$. aeruginosa control strains.

Funding information This work was funded by the Swiss National Science Foundation (projects number FNS-407240_177381 and FNS407240_177382).

\section{Compliance with ethical standards}

Conflict of interest The authors declare that they have no conflict of interest.

Ethical approval Not needed.

Informed consent All authors agreed on the submitted version. 


\section{References}

1. Boucher HW, Talbot GH, Bradley JS, Edwards JE, Gilbert D, Rice LB, Scheld M, Spellberg B, Bartlett J (2009) Bad bugs, no drugs: no ESKAPE! An update from the Infectious Diseases Society of America. Clin Infect Dis 48(1):1-12

2. Fernandez Barrat L, Ferret M, De Rosa F, Gabarrus A, Esperatti M, Terraneo S, Rinaudo M, Bassi L, Torres A (2017) Intensive care unit-acquired resistance due to Pseudomonas aeruginosa with and without multidrug resistance. J Inf Secur 74(2):142-152

3. Vincent JL, Rello J, Marshall J, Silva E, Anzueto A, Martin CD, Moreno R, Lipman J, Gomersall C, Sakr Y, Reinhart K (2009) International study of the prevalence and outcomes of infection in intensive care units. JAMA 302(21):2323-2329

4. Wright H, Bonomo RA, Paterson DL (2017) New agents for the treatment of infections with gram-negative bacteria: restoring the miracle or false dawn? Clin Microbiol Infect 23(10):704-712

5. Shin B, Park W (2017) Antibiotic resistance of pathogenic Acinetobacter species and emerging combination therapy. J Microbiol 55(11):837-849

6. Sievert DM, Ricks P, Edwards JR, Schneider A, Patel J, Srinivasan A, Kallen A, Limbago B, Fridkin S, National Healthcare Safety Network (NHSN) Team and Participating NHSN Facilities (2013) Antimicrobial-resistant pathogens associated with healthcareassociated infections: summary of data reported to the National
Healthcare Safety Network at the Centers for Disease Control and Prevention, 2009-2010. Infect Control Hosp Epidemiol 34(1):1-14

7. Willyard C (2017) The drug-resistant bacteria that pose the greatest health threats. Nature 543(7643): 15

8. Velkov T, Roberts KD, Nation RL, Thompson PE, Li J (2013) Pharmacology of polymyxins: new insights into an "old" class of antibiotics. Future Microbiol 8(6):711-724

9. EUCAST (2016) http://www.eucast.org/fileadmin/src/media/PDFs/ EUCAST_files/General_documents/Recommendations_for_MIC determination_of_colistin_March_2016.pdf

10. Nordmann P, Jayol A, Poirel L (2016) Rapid detection of polymyxin resistance in Enterobacteriaceae. Emerg Infect Dis 22(6):10381043

11. Lescat M, Poirel L, Tinguely C, Nordmann P (2019) A resazurin reduction-based assay for rapid detection of polymyxin resistance in Acinetobacter baumannii and Pseudomonas aeruginosa. J Clin Microbiol 57(3):e01563-e01518

12. Jayol A, Nordmann P, Lehours P, Poirel L, Dubois V (2017) Comparison of methods for detection of plasmid-mediated and chromosomally encoded colistin resistance in Enterobacteriaceae. Clin Microbiol Infect 24(2):175-179 